\title{
Efficient demosaicing through recursive filtering
}

\author{
Brice Chaix de Lavarène*, David Alleysson ${ }^{\dagger}$, Barthélémy Durette*, Jeanny Hérault* \\ *Image and Signal Laboratory, Joseph Fourier University, \\ Grenoble, France. Email: brice.chaix @ lis.inpg.fr \\ $\dagger$ Psychology and Neuro-Cognition Laboratory, Pierre Mendès France University \\ Grenoble, France. Email: david.alleysson@upmf-grenoble.fr
}

\begin{abstract}
We present a computationally efficient demosaicing algorithm based on a luminance-chrominance model of the Color Filter Array (CFA) image. We show that the chrominance information can be estimated using simple low-pass filtering. This algorithm allows us to use separable recursive filters, which are particularly adapted for real-time processing. Moreover, while most of demosaicing algorithms are specific to a particular CFA (usually the popular Bayer CFA), our method can be applied to any CFA. We present a linear version of the algorithm and an adaptive extension.
\end{abstract}

\section{INTRODUCTION}

Color demosaicing refers to the operation of constructing a color image from a mosaic of chromatic samples. In digital cameras today, the color information is sampled with a single sensor in front of which is placed a Color Filter Array (CFA). The resulting image has a single color value per pixel and should be interpolated to retrieve the corresponding color image. The interpolation is not perfect and there may appear artifacts in the reconstructed image. The most used CFA is the one proposed by Bayer [1].

Two main types of methods have been proposed in the literature : linear ones and adaptive ones, each corresponding to one characteristic of color images [2]. Linear methods exploit the spectral correlation or inter-plane correlation (the color planes are very similar one to each other in high frequencies). Since this correlation depends only on the spectral sensitivities of the color filters, it is assumed constant over the image and a linear uniform algorithm can be used. Nonlinear or adaptive methods exploit the spatial correlation or intra-plane correlation (there is a stronger correlation between pixels along a contour than across it). One assumes that a contour in the image should be treated differently than a uniform region. In particular these methods prevent from interpolating across edges.

The visual artifacts of the demosaicing process can be substantially reduced by taking into account both correlations. However, an adaptive algorithm means analyzing the image content, and hence increasing the computational complexity of the algorithm. At the opposite, linear methods are computationally efficient, but there may remain some artifacts in areas of high frequency content. Thus, demosaicing always results in a compromise between image quality and computation time. The seek for the optimal tradeoff is becoming more and more determining with the advent of cameras on embedded systems such as mobile phones. In this context, we propose a linear method which uses recursive filtering to reduce time of computation. We also propose an extension with adaptive processing to increase the quality of the reconstructed image.

Alleysson et al. have introduced a spectral model [3] that shows that a CFA image (or a mosaic image) is a spatial multiplexing of chromatic samples which modulates color information in spatial frequency while leaving luminance band based. A linear, space-invariant demosaicing algorithm was proposed from this model using a frequency selection approach to estimate luminance and chrominance. There are three drawbacks to this method. First, while the method is extremely simple, the high-order FIR (typically $7 \times 7$ ) is time consuming. Second, the method is not adaptive, and a zipper effect may arise along edges. And third, its luminance filter is specific to the Bayer CFA.

Adaptive methods also have a drawback in making the adaptive decision using the three color channels simultaneously. This makes the adaptive method dependent on the arrangement of the chromatic samples in the mosaic and prevents from using recursive filters. To our knowledge, the only attempts of demosaicing a general mosaic can be found in [2] and [4], but they are computationally heavy.

In the proposed method, we first estimate an alias-free, low-frequency (LF) version of the luminance signal in the CFA image. This LF luminance is substracted to the mosaic image, to obtain the high-frequency (HF) mosaic image. We show that if this HF mosaic image is demultiplexed, the chrominance signals are located at low frequency, where the LF luminance was previously removed from. This property allows us to estimate the chrominance signals using a simple low-pass filter. There are three advantages to the method. First, each step of the method is independent from the spatial arrangement of the chromatic filters. Second, the filters used are space-invariant and separable. An efficient implementation can be made using separable recursive filtering. Third, the LF luminance can be used to render the chrominance filtering adaptive to the luminance content (as other adaptive methods do) while still being independent from the configuration of the mosaic.

The paper is organised as follows. We first recall the spectral model in Section II. Then, we describe our new method in Section III. We finally discuss its implementation in both linear and adaptive manners, using recursive filters (Section IV). 
each chromatic channel of the image into three components. The pixel with no color information are set to zero, according to the $m_{i}$ patterns. In practice, it means selecting all the pixels of a chromatic class and copying them onto a matrix filled with zeros. This is formally equivalent to multiplying the mosaic image $I_{m}^{H F}$ by the submosaics $m_{i}$ :

$$
\left\{I_{m}^{H F}\right\}_{i}(x, y)=I_{m}^{H F}(x, y) m_{i}(x, y)
$$

Using Eqn 10, it comes:

$$
\left.\left\{I_{m}^{H F}\right\}_{i}\right)=I_{m} m_{i}-\phi^{L F} m_{i}
$$

where in fact $I_{m} m_{i}=C_{i} m_{i}$, and $C_{i}=\phi+\{\psi\}_{i}$. So we have:

$$
\begin{aligned}
\left\{I_{m}^{H F}\right\}_{i} & =\left(\phi+\psi_{i}\right) m_{i}-\phi^{L F} m_{i} \\
& =\left(\phi^{H F}+\psi_{i}\right) m_{i}
\end{aligned}
$$

The submosaic $m_{i}$ can be decomposed into the sum of a constant part $p_{i}$ and a modulating part $\tilde{m}_{i}, m_{i}=p_{i}+m_{i}$ as in Eqn. 2, which yields to:

$$
\left\{I_{m}^{H F}\right\}_{i}=\underbrace{p_{i}\left(\phi^{H F}+\psi_{i}\right)}_{\text {baseband }}+\underbrace{\left(\phi^{H F}+\psi_{i}\right) m_{i}}_{\text {modulated }}
$$

We thus have two terms in the expression of $\left\{I_{m}^{H F}\right\}_{i}$ : one term is baseband, the other one is HF modulated (Fig. 2(b)). Moreover, if the frequency cutoff $f_{c}$ of filter $f$ was chosen to match the chrominance bandwidth, $I_{m}^{H F}$ and $\psi_{i}$ have disjoint supports (the chrominance of natural images $\psi$ beeing generally a lowpass signal). Consequently, a simple lowpass filter on the demultiplexed HF image is sufficient to recover the full chrominance components of the image. In practice we use the same filter $f$ used for the estimation of $\phi^{L F}$ :

$$
\{\psi\}_{i}=\frac{1}{p_{i}} f *\left\{I_{m}^{H F}\right\}_{i}
$$

Knowing the mosaic image and the chrominance components, one can retrieve the full luminance component by substraction:

$$
\phi=I_{m}-\sum_{i} \psi_{i} m_{i}
$$

An error-free recovery is theoretic, in practice there is aliasing between chrominance and modulated luminance which expresses itself through false colors and/or zipper noise. To improve the visual rendering, an adaptive extension of the algorithm will be introduced in the next section.

To sum up, the methods can ben divided into 5 steps:

1) separation of the mosaic image $I_{m}$ into lowpass $\left(I_{m}^{L F}=\right.$ $\left.\phi^{L F}\right)$ and highpass $\left(I_{m}^{H F}\right)$ components using a lowpass filter $f$,

2) demultiplexing of the HF component,

3) using $f$, filtering of each marginal plane of the demultiplexed image in order to get the chrominance components $\psi_{R}, \psi_{G}$ and $\psi_{B}$,

4) retrieving of the luminance component $\phi$ by substraction between the mosaic image and the "remodulated" chrominance $^{1}$,

\footnotetext{
${ }^{1}$ the modulation is purely virtual, since it consists in discarding some pixels according to the zeros in the lattice $m_{i}$
}

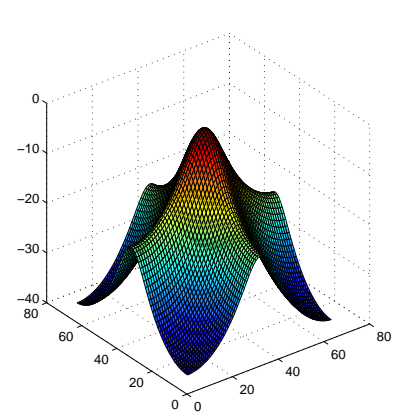

Fig. 3. Frequency response of the IIR filter (dB).

5) addition of luminance to chrominance to get an $C_{R}, C_{G}$ and $C_{B}$.

\section{IMPLEMENTATION}

In the following section, we present two implementations of the approach that was described in the preceding section. Both use recursive filtering, which makes them particularly fast. One is a linear version whereas the other one is an edgeadaptive extension.

\section{A. Linear method}

The crucial point of the linear method is the design of the filter $f . f$ has the only constraint to be lowpass, and to match the chrominance bandwidth. For computational efficiency, we chose to implement it using a separable recursive filter whose $z$-transform is:

$$
F\left(z_{1}, z_{2}\right)=F_{1}\left(z_{1}\right) F_{2}\left(z_{2}\right)
$$

where $z_{1}$ and $z_{2}$ are the horizontal and vertical variables, and with

$$
F_{k}\left(z_{k}\right)=\frac{1-a}{1+a} \frac{1}{1-a z_{k}^{-1}} \frac{1}{1-a z_{k}}
$$

Note that $F$ involves only 4 neighbors. Its frequency response is represented in Fig. 3. As the reader can see, $\mathrm{F}$ attenuates much more in diagonal than in horizontal/vertical directions. Hence, a one-order numerator is needed for mosaics whose $m_{i}$ modulate at horizontal or vertical direction (e.g. the Bayer pattern for $\mathrm{R} / \mathrm{B}$ channels) in order to vanish the filter at frequencies $(0, \pm 0.5)$ and $( \pm 0.5,0)$. However, this FIR is superfluous for CFA's that do not modulate chrominance in horizontal and vertical directions at the Niquist frequency (i.e. $0.5)$.

The parameter $a$ controls the cutoff frequency, and hence, the tradeoff between false colors and zipper noise. For the Kodak database, we empirically found $a=0.5$.

Note that Eqn. 16, the densities $p_{i}$ of the color filters are involved. For the Bayer CFA, these are constant and have values $\{0.25,0.5,0.25\}$. In a general case, these densities may vary locally around the global mean values. These variations have to be taken into account in the filtering process in order 

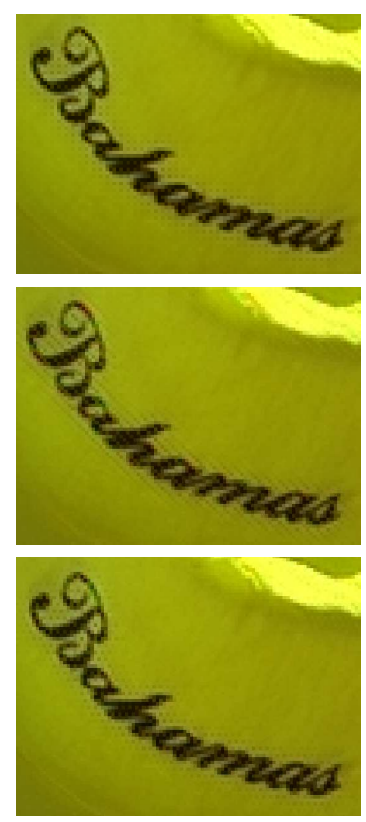

(a)
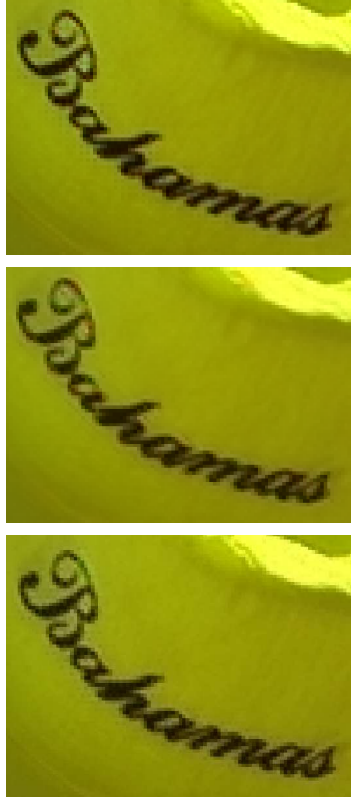

(b)

Fig. 4. Crop of a demosaiced image using: (a) (b) .

to match the luminance definition (Eqn. 6). Eqn. 16 will be thus rewritten (for $i \in\{R, G, B\}$ ):

$$
\psi_{i}=\frac{f *\left\{I_{m}^{H F}\right\}_{i}}{f * m_{i}}
$$

This technique is called normalised convolution [7]. Note that $f * m_{i}$ depends only on the mosaic and can thus be hard coded. The same precaution holds for the estimation of $\phi^{L F}$.

\section{B. Adaptive extension}

The coarse, alias-free luminance $\phi^{L F}$ can be exploited in an edge-adaptive manner. This "rough" estimate may appear suboptimal to the reader who is aware that classical demosaicing methods use the mosaic image $I_{m}$ directly (e.g. [5], [8]), without any lowpass filtering. However these methods must compute gradients between pixels of the same class $(\mathrm{R}, \mathrm{G}$ or B), wich are not adjacent on the CFA. Therefore, $\phi^{L F}$ contains the same amount of spatial information as the marginal planes of the mosaic image do. Moreover $\phi^{L F}$ has the great advantage of bein totally independent from the mosaic, since it contains pure spatial information. The edge detection is thus mosaic blind. We compute the horizontal $w_{1}$ and vertical $w_{2}$ gradients ( $w_{2}$ has the same definition as $w_{1}$, but transposed to the vertical direction):

$$
w_{1}(x, y)=\operatorname{abs}\left(\phi^{L F}(x, y+1)-\phi^{L F}(x, y-1)\right)
$$

These gradients dictates the choice of either $F_{1}$ or $F_{2}$ at each pixel for the direction of the chrominance filtering. As other adaptive demosaicing methods, a postprocessing step is needed, in order to update the values of chrominance with the estimated luminance.

\section{Results}

The linear version has the same visual performance in term of quality than in [9], as shown by Fig. 4(a), but drastically reduces the computation time, because of the recursive filter. Our estimate of the equivalent FIR would be of size $9 \times 9$. The adaptive method substantially removes the zipper noise that arises with the linear method (Fig. 4(b)). It is noteworthy that the CFA pattern is recognizable through the zipper noise. The artifact is thus more or less perceivable depending on the pattern.

\section{CONCLUSION}

We presented a new demosaicing approach that has the advantage to be efficient in computational time and to be applicable to any CFA arrangement, while giving a visual result comparable to the most recent methods. In this implementation, the coefficient of the recursive filtering is constant over the image. It would be interesting to study the effect of an adaptive coefficient that follows the content of the image.

Finally, this algorithm makes an analogy with the visual system. The human retina provides a coarse estimate of the achromatic spatial information in a channel (in the output of the retina) called magno-cellular pathway. This channel is thought to prepare the information for the brain before the arrival of the details of spatial information (high frequencies of luminance) and color oppositions, which are conveyed by the parvo-cellular pathway.

\section{REFERENCES}

[1] B. Bayer, "Color imaging array," US patent 3,971,065, to Eastman Kodak Company, 1976.

[2] R. Lukac and K. N. Plataniotis, "Universal demosaicking for imaging pipelines with an rgb color filter array," Pattern Recognition, vol. 38, pp. 2208-2212, November 2005.

[3] D. Alleysson, S. Süsstrunk, and J. Hérault, "Linear color demosaicing inspired by the human visual system," IEEE Transactions on Image Processing, vol. 14, pp. 439-449, April 2005.

[4] W. A. Zhu, K. J. Parker, and M. A. Kriss, "Color filter arrays based on mutually exclusive blue noise patterns," in Proc. SPIE Vol. 3300, p. $207-$ 218, Color Imaging: Device-Independent Color, Color Hardcopy, and Graphic Arts III, Giordano B. Beretta; Reiner Eschbach; Eds., G. B. Beretta and R. Eschbach, Eds., Jan. 1998, pp. 207-218.

[5] N. Lian, L. Chang, and Y.-P. Tan, "Improved color filter array demosaicking by accurate luminance estimation," in IEEE International Conference on Image Processing, vol. 1, September 2005, pp. 41-44.

[6] E. Dubois, "Frequency-domain methods for demosaicking of bayersampled color images," IEEE Signal Processing Letters, vol. 12, pp. 847850, December 2005

[7] H. Knutsson and C.-F. Westin, "Normalized and differential convolution," in Computer Vision and Pattern Recognition, 1993. Proceedings CVPR '93., 1993 IEEE Computer Society Conference on, 15-17 June 1993, pp. 515-523.

[8] K. Hirakawa and T. W. Parks, "Adaptive homogeneity-directed demosaicing algorithm," IEEE Transactions on Image Processing, vol. 14, no. 3, pp. 360-369, December 2005.

[9] B. C. de Lavarène, D. Alleysson, and J. Hérault, "Practical implementation of lmmse demosaicing using luminance and chrominance spaces," Computer Vision and Image Understanding, Special Issue on Color Image Processing, In Press 2007. 